Document downloaded from:

http://hdl.handle.net/10251/152481

This paper must be cited as:

Zheng, D.; Madrigal-Madrigal, J.; Barrera Vilar, D.; Sales Maicas, S.; Capmany Francoy, J. (2017). Microwave Photonic Filtering for Interrogating FBG-Based Multicore Fiber Curvature Sensor. IEEE Photonics Technology Letters. 29(20):1707-1710.

https://doi.org/10.1109/LPT.2017.2742579

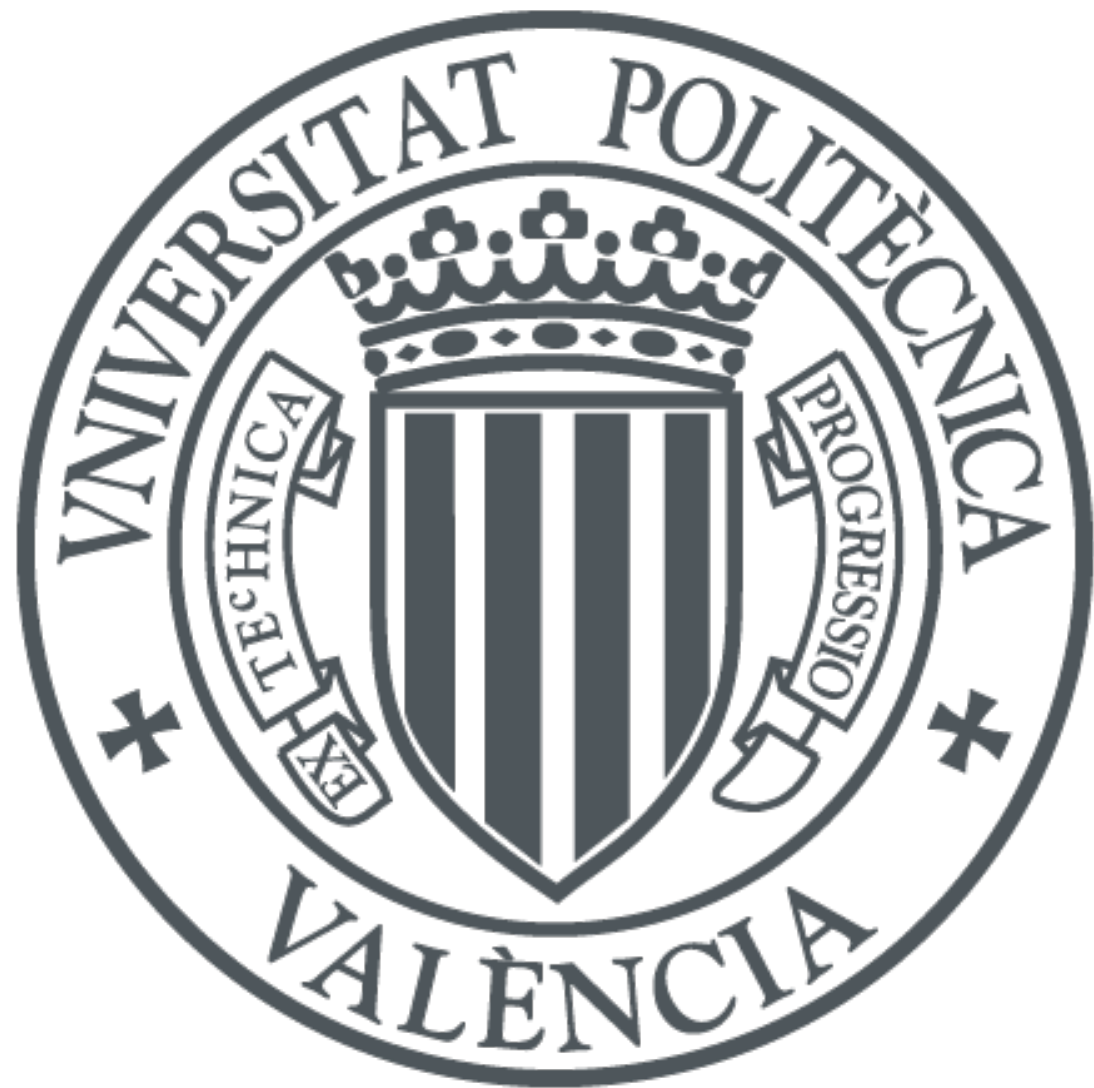

The final publication is available at

https://doi.org/10.1109/LPT.2017.2742579

Copyright Institute of Electrical and Electronics Engineers

Additional Information

"(C) 2017 IEEE. Personal use of this material is permitted. Permissíon from IEEE must be obtained for all other uses, in any current or future media, including reprinting/republishing this material for advertisíng or promotional purposes, creating new collective works, for resale or redistribution to servers or lists, or reuse of any copyrighted component of this work in other works." 


\title{
Microwave Photonic Filtering for Interrogating FBG-based Multicore Fiber Curvature Sensor
}

\author{
D. Zheng, J. Madrigal, D. Barrera, S. Sales, Senior Member, IEEE, and J. Capmany, Fellow, IEEE
}

\begin{abstract}
We propose and experimentally demonstrate an approach to perform high-resolution and temperature-insensitive interrogation of a fiber Bragg grating (FBG) based multicore fiber curvature sensor using a microwave photonics filtering technique. By combining two reflected sample signals from the two FBGs inscribed in multicore fiber (MCF) and a dispersion device, a twotap notch microwave photonic filter (MPF) is formed. The notch frequency of MPF is dependent on the time delay difference between the two FBG reflected signals, which is related to wavelength spacing of the two FBGs. Due to the wavelength spacing of the two FBGs is proportional to the curvature, the curvature can be readily interrogated by monitoring the notch frequency of MPF. The proposed interrogation scheme with the sensitivity of $92 \mathrm{MHz} / \mathrm{m}^{-1}$ is achieved, whilst the sensitivity can be easily adjusted. Moreover, the proposed interrogation scheme is temperature-insensitive.
\end{abstract}

Index Terms-Optical fiber sensors, fiber Bragg gratings, curvature interrogation, microwave photonic filter.

\section{INTRODUCTION}

$\mathrm{M}$ ulticore fibers (MCFs) have been widely investigated during the last few decades. Benefiting from the unique property of featuring more than one core within a single cladding, MCFs have attracted considerable research interest in the applications such as high-capacity optical transmission networks and microwave photonics systems to cope with the exponential growth of data transmission demand [1-3]. Moreover, MCFs have also attracted much attention by optical sensing community due to its distinctive characteristics of compactness, easy multiplexing as well as structural diversity. MCFs based sensors such as curvature/bending [4,5], shape [6], flow velocity [7], accelerometers sensing [8] and chemical sensing $[9,10]$ have been reported and widely used in various applications like mechanical engineering and structural health monitoring.

Because of their intrinsic characteristics, MCFs are particularly well suited for curvature/bending sensing. Generally, MCFs based curvature sensors can be classified into two main categories: FBGs-assisted sensor and interferometertype sensor. The first demonstration of MCFs based curvature

This paragraph of the first footnote will contain the date on which you submitted your paper for review. This work was supported in part by the National Natural Science Foundation of China (61405166) and the China Scholarship Council, in part by Generalitat Valenciana (APOSTD/2016/015, GVA PROMETEO 2013/012); Ministerio de Economía y Competitividad (MINECO) (TEC2014-60378-C2-1-R). sensor consisting of two FBGs inscribed in a four-core fiber was reported by Gander et al. in 2000 [11]. Soon afterwards, the two-axis curvature sensor was verified by writing three FBGs into three separate cores of a multicore fiber [12]. In order to realize dynamic curvature measurement, arrayed waveguide gratings have been adopted to demodulate multicore FBGs curvature sensor [13]. Apart from single-point measurement, we have proposed a multipoint two-dimensional curvature sensor by inscribing arrays of apodized highly reflective FBGs in nontwisted homogeneous four-core fiber [5].

On the other hand, interference structures are another wellestablished way to design MCFs based curvature sensor. The simplest way is to use a length of multicore fiber as a bending sensor, the bending angle can be calculated using phase values derived from Fourier analysis of the far-field interferogram [14]. Alternatively, super-mode interference in MCFs can also be utilized to achieve compact curvature sensor, the bending sensitivity is as high as $3000 \mathrm{~nm} / \mathrm{mm}^{-1}$ [15]. In addition, a type of combination structures consisting of single mode fiber, multimode fiber and MCFs has emerged as a high-performance bending sensor solution $[16,17]$.

Among the various structures mentioned above, FBG-based MCFs curvature sensors have the simplest structure and interrogation scheme. However, the previously reported interrogation techniques tend to rely on the use of wavelength measurement and optical power detection. The wavelengthbased interrogation schemes are implemented by using an optical spectrum analyzer (OSA) to measure the center wavelength of FBG or the peaks/dips of the interference spectrum. Therefore, the interrogation speed is rather slow and the resolution is strongly restricted by the OSA's performance. In the case of an optical-power-based interrogation scheme, an optical filter or optical interferometer is employed for ratiometric detection to identify the wavelength shift of FBG. Nevertheless, this kind of approach is very sensitive to ambient fluctuation. Any frequency deviations of the optical filter or interferometer induced by temperature fluctuation or strain changes will result in interrogation errors.

Recently, microwave photonic technique for interrogation of

Di Zheng is with the Center for Information Photonics \& Communications, School of Information Science \& Technology, Southwest Jiaotong University, Chengdu, 610031, China (e-mail: dzheng@home.swjtu.edu.cn ).

Javier Madrigal, David Barrera, Salvador Sales and Jose Capmany are with Photonics Research Labs, ITEAM Research Institute, Universitat Politècnica de València, Camino de Vera, s/n, 46022, Valencia, Spain (e-mail: jamadmad@iteam.upv.es, dabarvi@,iteam.upv.es, ssales@dcom.upv.es , jcapmany@iteam.upv.es ). 
FBG based sensor has attracted extensive interest owing to its high resolution and distributed demodulation capability [18], [19]. In this paper, a microwave photonics filtering technique is proposed to interrogate a FBG-based MCF curvature sensor for the first time, to the best of our knowledge. The basic principle exploited for the proposed interrogation scheme is based on a two-tap notch MPF, which is implemented by two reflected signals from two FBGs inscribed in MCF in conjunction with a dispersion device. Due to the notch frequency of the MPF is determined by the wavelength separation of the two FBGs, which is related to the applied curvature, thus the curvature can be readily interrogated by detecting the notch shift of the MPF. Furthermore, the proposed interrogation scheme limits the effect of ambient fluctuations.

\section{PRINCIPLE}

The proposed FBG based MCF curvature sensing system is illustrated in Fig.1. The output from an incoherent broadband light source (BBS) is coupled into an electric-optical modulator (EOM), where the optical signal is modulated by a microwave signal generated from a vector network analyzer (VNA). Then the modulated optical signal is sent into two FBGs inscribed separately at two outer cores of a MCF through a fan-in/fan-out device. Subsequently, the reflected signals from the two FBGs pass through the fan-in/fan-out device again and combined at an optical coupler (OC). Finally, the combined optical signal is detected by photodiode (PD) after a dispersive device and corresponding frequency response is measured by VNA. A tunable delay line (TDL) is used to provide a fixed time delay difference (TDD) between the two reflected signals, a variable TDD induced by a length of dispersion compensating fiber (DCF) is determined by the wavelength spacing of the two FBGs. Since the two reflected signals undergo different time delays, a two-tap notch MPF is thus realized. When the plane of two FBGs aligns to the bending direction, the inner side of the bending fiber will be compressed while the outer side will be stretched, such that the wavelength separation of two FBGs increases and the total TDD between the two reflected signals is varied. In order to obtain the maximum notch depth, a variable optical attenuator (VOA) is adopted to balance the optical power of the two reflected sample signals.

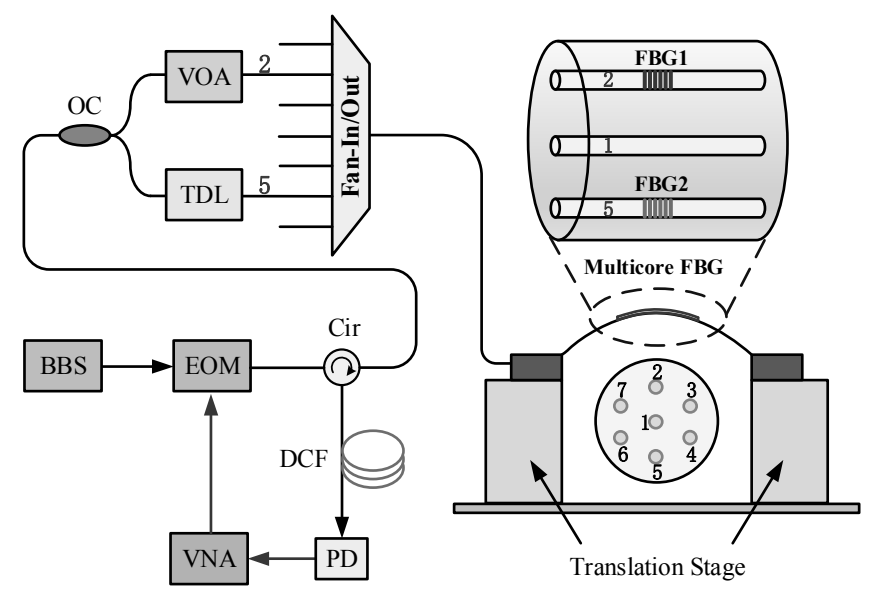

Fig.1. Schematic of the proposed curvature sensing system.

The working principle is explained as follows: The electrical response $H(\Omega)$ of the proposed interrogation scheme is essentially a two-tap notch MPF, which is given by [20]:

$$
H(\Omega)=a_{0}+a_{1} e^{-j \Omega T}
$$

where $a_{0}$ and $a_{1}$ are the weight of the two taps, which are related to the reflected power of the two FBGs. The total TDD, $T$, between two samples is made up of two parts:

$$
T=T_{0}+D \Delta \lambda
$$

where $T_{0}$ is the fixed TDD introduced by the TDL, and the second term is the tunable TDD induced by the dispersion device. $D$ is the value of the group velocity dispersion ( $\mathrm{ps} / \mathrm{nm})$ of the DCF. The wavelength difference between the two FBGs caused by the applied curvature $C$ is given by [9]:

$$
\Delta \lambda=\lambda_{B}\left(1-p_{\varepsilon}\right) d C
$$

where $\lambda_{B}$ is the initial wavelength when no bending is applied on $\mathrm{MCF}, p_{\varepsilon}$ denotes the effective photo-elastic coefficient with a typical value of 0.22 in silica fiber, $d$ is the distance between the two FBGs. The notches of the MPF are given by:

$$
f_{n}^{\text {notch }}=\left(n+\frac{1}{2}\right) \frac{1}{T}, \quad n=0,1,2 \ldots
$$

where $1 / T$ is equal to the free spectrum range (FSR) of the MPF and $n$ represents the $n$th notch resonance. By substituting (2) and (3), (4) can be rewritten as;

$$
f_{n}^{\text {notch }}=\left(n+\frac{1}{2}\right)\left(T_{0}+A C\right)^{-1}
$$

where $A=D \lambda_{B}\left(1-p_{\varepsilon}\right) d$. When multicore fiber is curved, the wavelength spacing of the two FBGs changes, and accordingly, $T$ is varied. By evaluating the notch positions, the interrogation of FBG-based MCF curvature sensor is achieved.

The curvature setup is composed by one steel strip and two three axis translation stages. The MCF is placed at the top of the steel strip and the FBGs are located in the center of the steel strip. The plane of the two FBGs is parallel to the bending direction. When the two translation stages approach each other, the metal strip is bent and nearly uniform curvature are obtained. The applied curvature $C$ can be calculated by [21]:

$$
\sin \left(\frac{L C}{2}\right)=\frac{(L-\Delta L) C}{2}
$$

where $L$ is the length of the steel strip and $\Delta L$ corresponds to the distance that translations stage travels when we turn the micrometer screw.

\section{EXPERIMENT RESULTS AND DISCUSSION}

Experimental measurements have been performed using the setup illustrated in Fig. 1. A commercial seven-core MCF from Fibercore Ltd. is used for the implementation of the curvature sensing. The MCF has a cladding diameter of $125 \mu \mathrm{m}$ and the cores are arranged in a hexagonal pattern with one of the cores in the center, as seen in Fig.1. The core spacing is $35 \mu \mathrm{m}$ and each core has a mode field diameter of $6.4 \mu \mathrm{m}$ and a numerical 
aperture of 0.2 . In order to increase the photosensitivity, the MCF is hydrogen-loaded at ambient temperature for two weeks at 50 bar pressure. The arbitrary two outer cores arranged symmetric to the center core as well as the center core itself are selected to inscribe FBG simultaneously using a $244 \mathrm{~nm}$ $\mathrm{CW}$ frequency-doubled argon-ion laser; a detailed description of FBG inscription can be found in [3]. The fan-in/fan-out devices are provided by Optoscribe and have a maximum level of insertion loss of $2.5 \mathrm{~dB}$ in each direction, including additional $1 \mathrm{~dB}$ losses due to the MCF splices.

Fig.2 shows the measured reflection spectra of the three FBGs inscribed in three different cores. FBG1 and FBG2 written in the two outer cores have almost same FWHMs of 0.2 $\mathrm{nm}$ and the central wavelength difference of $\sim 0.2 \mathrm{~nm}$. An extra FBG3 inscribed in central core plays the role of monitoring externally applied axial strain during the bending experiment, make sure that the two outer core FBGs only experience bendinduced strain.

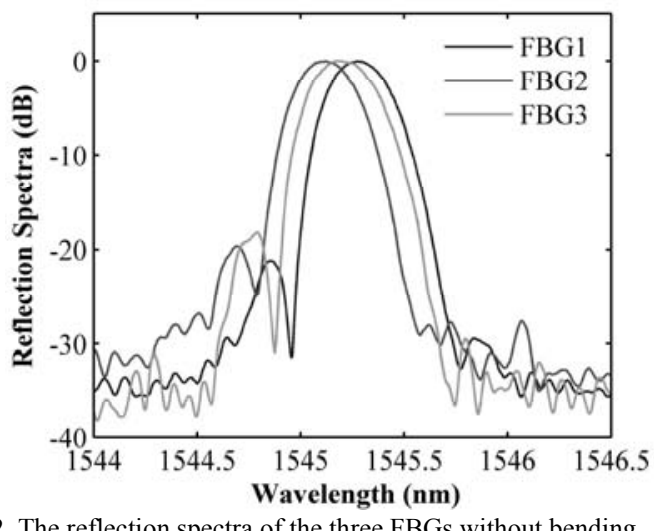

Fig.2. The reflection spectra of the three FBGs without bending.

During the bending experiment, the $T_{0}$ induced by the TDL is a constant with a value of 315 ps. The DCF used in our experiment has a total dispersion parameter of $-203 / \mathrm{ps} / \mathrm{nm}$. Fig. 3 shows the measured frequency response of the two-tap notch MPF at different curvature. Due to the TDD introduced by the TDL is fixed, thus the frequency shift of the notch position is only determined by the tunable TDD induced by DCF, which is related to the wavelength spacing between FBG1 and FBG2. In our experiment, the FBG1 is located at the outer

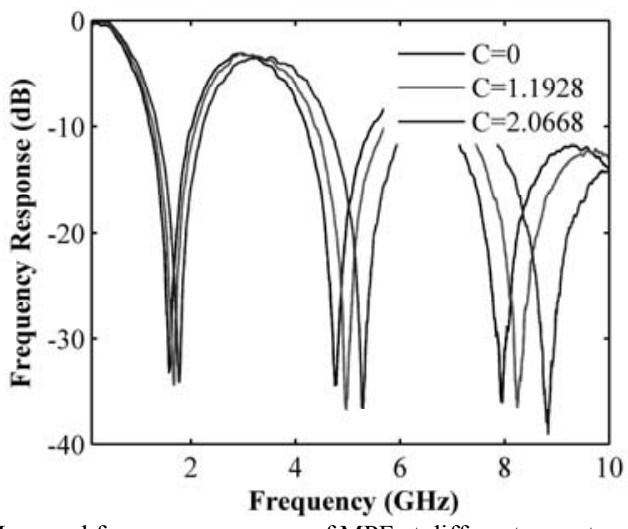

Fig.3. Measured frequency response of MPF at different curvature. side of the bending fiber while FBG2 is placed at the inner side, so the notch position increases with increasing curvature, which indicates that the wavelength spacing is increased as the curvature increases.

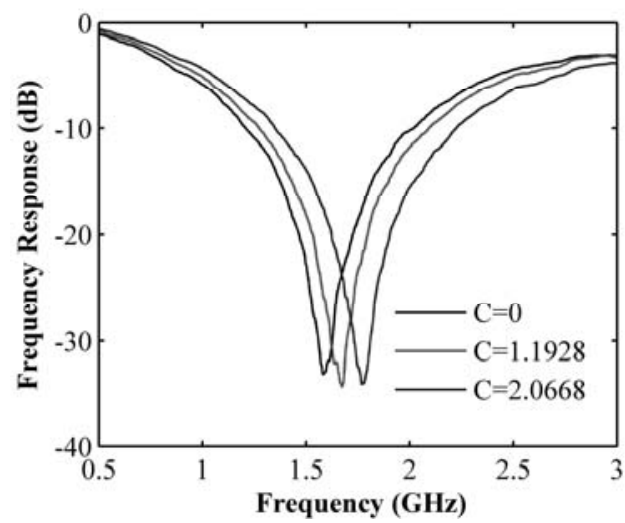

Fig. 4. Zoom-in views of the first notch at different curvature.

Fig. 4 shows a zoom-in view of the first notch frequency illustrated in Fig.3. It should be noted that, during the whole bending process, the notch depth is always larger than $30 \mathrm{~dB}$, so high resolution for curvature sensing can be achieved. When no bending is applied on the MCF, the first-notch frequency is located around $1.582 \mathrm{GHz}$, which agrees well with the theoretical value of $1.587 \mathrm{GHz}$ calculated by (5).

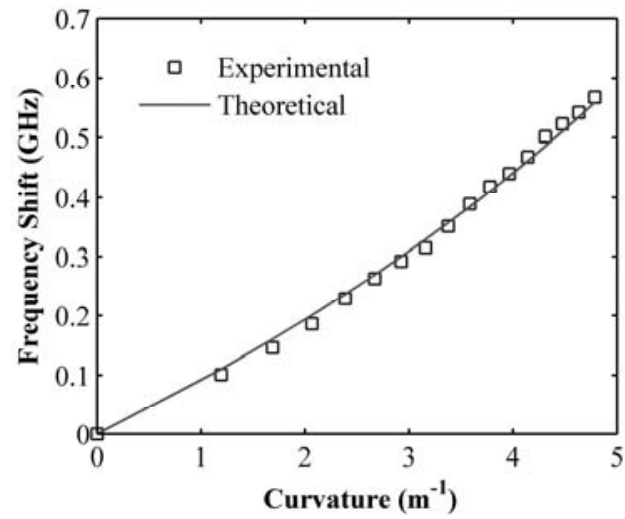

Fig. 5. The relationship between the curvature and the first-notch frequency shift.

Because the interrogation speed is determined by the scanning range of the VNA, the first-notch frequency is used to perform the sensing measurement in order to increase the interrogation speed. Fig. 5 shows the measured first-notch frequency shift as a function of the curvature. The experimental result is in good agreement with the theoretical result. It should be noted that the sensitivity is increased gradually with the curvature. Even in the lower curvature, the sensitivity is still as high as $92 \mathrm{MHz} / \mathrm{m}^{-1}$, thus, our proposed scheme allows a better discrimination at the lower curvature in comparison to conventional interrogation schemes. Thanks to the ultrahigh measurement resolution $(\sim 1 \mathrm{~Hz})$ of the VNA, the resolution of the proposed interrogation scheme can reach $9.2 \times 10^{-5} \mathrm{~m}^{-1}$ in theory for a given TDD parameters.

It should be pointed out that the sensitivity can be easily 
enhanced by adopting longer DCF or chirp fiber grating with a large dispersion value, or by decreasing the fixed TDD. In addition, due to the periodicity of the MPF, the higher order notch frequency can be used as monitoring points to enhance the sensitivity. In theory, if the $k$ th notch is chosen as a sensing point, its sensitivity will be $(2 k+1)$ times of that of the first notch frequency.

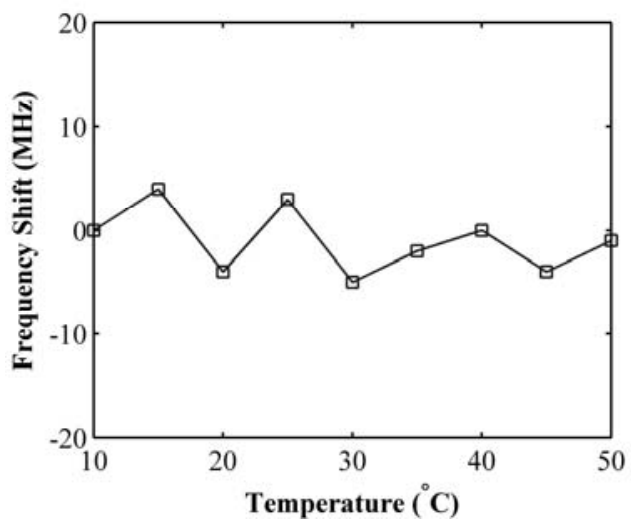

Fig. 6. Measured frequency change of the first-order notch against temperature.

A test to verify the temperature-insensitivity was also carried out, as shown in Fig. 6. Here the temperature was turned from 10 to $50{ }^{\circ} \mathrm{C}$ with a step of $5{ }^{\circ} \mathrm{C}$. As can be seen, the frequency shift variations is as small as just less than $5 \mathrm{MHz}$, and we attributed these tiny changes to the small background dispersion fluctuation of DCF. Because the two FBGs are written at the same position along the length of the MCF, they will experience the same temperature-dependent wavelength shift. Therefore, the wavelength spacing of the two FBGs will not be altered and, accordingly, the notch frequency exhibits high stability. It is also worth noting that, besides temperature-insensitivity, the proposed interrogation method could be almost insensitive to many others random perturbations including polarization drifts and power fluctuation of BBS. This excellent robustness feature is highly attractive for remote interrogation from long distance and/or harsh environments.

\section{CONCLUSION}

In conclusion, a novel interrogation scheme exploiting microwave photonics filtering technique for FBG-based MCF curvature sensor is proposed and experimentally demonstrated. The fundamental principle of the proposed scheme is based on a two-tap notch MPF, which is implemented by two reflected signals from two FBGs inscribed in MCF in conjunction with a dispersion device. The notch frequency of the MPF with respect to curvature are obtained, and the sensitivity is larger than 92 $\mathrm{MHz} / \mathrm{m}^{-1}$. Thanks to the high resolution of an electrical spectrum analyzing equipment, the curvature can be precisely interrogated. Additionally, the interrogation scheme is insensitive to temperature fluctuation.

\section{REFERENCES}

[1] B. Zhu, et al., "112-Tb/s Space-division multiplexed DWDM transmission with $14-\mathrm{b} / \mathrm{s} / \mathrm{Hz}$ aggregate spectral efficiency over a $76.8-\mathrm{km}$ seven-core fiber," Opt. Express, vol. 19, no. 17, pp. 16665-16671, 2011.

[2] I. Gasulla and J. Capmany, "Microwave photonics applications of multicore fibers," IEEE Photon. J., vol. 4, no. 3, pp. 977-888, 2012.

[3] I. Gasulla, D. Barrera, J. Hervás and S. Sales, "Spatial division multiplexed microwave signal processing by selective grating inscription in homogeneous multicore fibers," Sci. Rep., vol. 7, pp. 41727, 2017.

[4] F. M. Araujo, L. A. Ferreira, and J. L. Santos, "Simultaneous determination of curvature, plane of curvature, and temperature by use of a miniaturized sensing head based on fiber Bragg gratings," Appl. Opt., vol. 41, no. 13, pp. 2401-2407, 2002.

[5] D. Barrera, I. Gasulla, and S. Sales, "Multipoint two-dimensional curvature optical fiber sensor based on a nontwisted homogeneous fourcore fiber," J. Lightw. Technol., vol. 33, no. 12, pp. 2445-2450, 2015.

[6] J. P. Moore and M. D. Rogge, "Shape sensing using multi-core fiber optic cable and parametric curve solutions," Opt. Express, vol. 20, no. 3, pp. 2976-2973, 2012

[7] L. B. Yuan, J. Yang and Z. H. Liu, "A compact fiber-optic flow velocity sensor based on a twin-core fiber Michelson interferometer," IEEE Sensors J., vol. 8, no. 7, pp. 1114-1117, 2008.

[8] A. Fender, et al., "Two-axis temperature-insensitive accelerometer based on multicore fiber Bragg gratings," IEEE Sensors J., vol. 8, no. 7, pp. 1292-1298, 2008

[9] S. J. Zheng, "Long-period fiber grating moisture sensor with nanostructured coatings for structural health monitoring," Struct. Health Monit., vol. 14, no. 2, pp. 148-157, 2014.

[10] S. J. Zheng, M. Ghandehari, J. P. Ou, "Photonic crystal fiber long-period grating absorption gas sensor based on a tunable erbium-doped fiber ring laser," Sensor Actuat. B-Chem, vol. 223, pp. 324-332, 2016.

[11]M. J. Gander, et al., "Bend measurement using Bragg gratings in multicore fiber," Electron. Lett., vol. 36, no. 2, pp. 120-121, 2000.

[12]G. M. H. Flockhart, et al., "Two-axis bend measurement with Bragg gratings in multicore optical fiber," Opt. Lett., vol. 28, no. 6, pp. 387-389, 2003.

[13]A. Fender, et al., "Dynamic two-axis curvature measurement using multicore fiber Bragg gratings interrogated by arrayed waveguide gratings," Appl. Opt., vol. 45, no. 36, pp. 9041-9048, 2006.

[14] M. J. Gander, et al., "Two-axis bend measurement using multicore optical fibre," Opt. Commun., vol. 182, pp. 115-121, 2000.

[15]G. S. Delgado, et al., "Compact fiber-optic curvature sensor based on super-mode interference in a seven-core fiber," Opt. Lett., vol. 40, no. 7, pp. 1468-1471, 2015.

[16]S. Wang, et al., "Two-dimensional bending vector sensor based on the multimode-3-core-multimode fiber structure," IEEE Photon. Technol. Lett., vol. 29, no. 10, pp. 822-825, 2017.

[17]Y. Zhao, L. Cai and X. G. Li, "Temperature-insensitive optical fiber curvature sensor based on SMF-MMF-TCSMF-MMF-SMF structure," IEEE Trans. Instrum. Meas., vol. 66, no. 1, pp. 141-147, 2017.

[18] A. L. Ricchiuti, J. Hervás, D. Barrera, S. Sales, and J. Capmany, "Microwave photonics filtering technique for interrogating a very-weak fiber Bragg grating cascade sensor," IEEE Photon. J., vol. 6, no. 6, pp. 5501410,2014

[19]L. Xia, R. Cheng, W. Li and D. M. Liu, "Identical FBG-based quasidistributed sensing by monitoring the microwave responses," IEEE Photon. Technol. Lett., vol. 27, no. 3, pp. 323-325, 2015.

[20] J. Capmany and D. Novak, "Microwave photonics combines two worlds," Nature Photon., vol. 1, pp. 319-330, 2007.

[21]H. L. Zhang, Z. F. Wu, P. P. Shum, R. X. Wang, X. Q. Dinh, S. N. Fu, W. J. Tong and M. Tang, "Fiber Bragg gratings in heterogeneous multicore fiber for directional bending sensing," J. Optics, vol. 18, pp. 085705, 2016. 